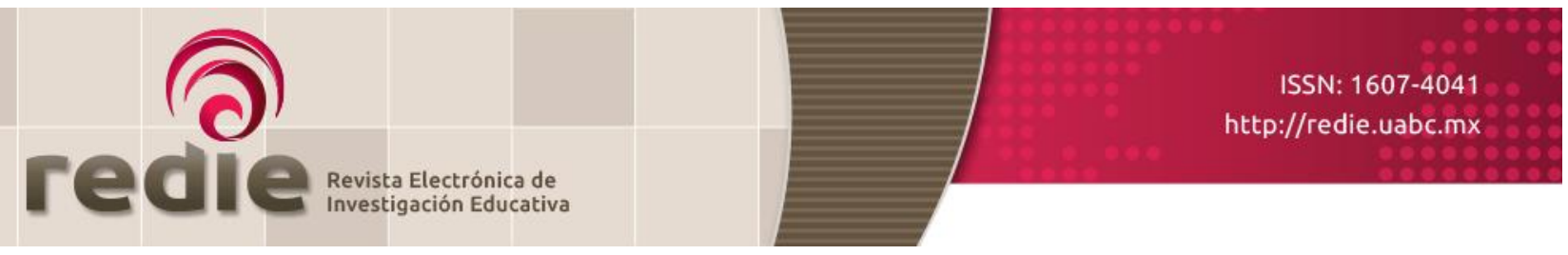

Vol. 20, Núm. 3, 2018

\title{
Desmotivación del alumnado de secundaria en la materia de matemáticas
}

\section{Demotivation in Mathematics among High School Secondary}

María-Carmen Ricoy (1) cricoy@uvigo.es

Maria João V.S. Couto (2) mjoaocouto@uvigo.es

(1) Universidad de Vigo

(2) Instituto Politécnico de Porto

(Recibido: 16 de octubre de 2016; Aceptado para su publicación: 13 de febrero de 2017)

Cómo citar: Ricoy, M-C y Couto, M. J. (2018). Desmotivación del alumnado de secundaria en la materia de matemáticas. Revista Electrónica de Investigación Educativa, 20(3), 69-79. https://doi.org/10.24320/redie.2018.20.3.1650

\section{Resumen}

El fracaso académico constituye una preocupación de la comunidad educativa. Por ello, asignaturas que cuentan con circunstancias poco favorables merecen una especial atención. El objetivo central de esta investigación es identificar y analizar los componentes de la desmotivación en los adolescentes en la materia de matemáticas en la Educación Secundaria. Este estudio se desarrolló desde un enfoque cualitativo, a través de un procedimiento introspectivo a partir de la percepción del profesorado. Para ello, se han realizado 70 entrevistas a docentes de Matemáticas de Portugal, de ellos 51 son mujeres y 19 varones. A las distintas narrativas obtenidas se le ha aplicado un análisis de contenido. Los resultados y conclusiones ponen de manifiesto, como principales integrantes de la desmotivación, la insuficiente formación del alumnado, así como algunas dinámicas tradicionales de enseñanza utilizadas por los docentes de Matemáticas. Además, cabe añadir una influencia negativa del ambiente.

Palabras clave: Desmotivación, educación secundaria, didáctica de las matemáticas.

\section{Abstract}

Academic failure is a concern in the education community; more taxing subjects therefore deserve special consideration. The primary objective of this study was to identify and analyze factors of adolescent demotivation in mathematics in high school. This study followed a qualitative approach, using an introspective process based on teachers' perceptions. To this end, 70 mathematics teachers from Portugal were interviewed, of whom 51 were female and 19 male. Content analysis was performed on the different narratives obtained. The results and conclusions show that the main constituents of demotivation are inadequate prior education and certain traditional teaching activities used by mathematics teachers. A negative influence from the environment should also be noted. 


\section{Introducción}

El estudio sobre la problemática de la desmotivación asociada a la disciplina de Matemáticas en enseñanza secundaria es de gran interés para la comunidad educativa y científica. En este sentido, Gómez-Chacón (2010) apunta que generar una mejor comprensión y significado matemático en la escuela conlleva un componente afectivo que requiere ser analizado y entendido.

Los componentes ligados con el afecto, las emociones, las actitudes e incluso las creencias pueden influenciar de forma provechosa el aprendizaje en la materia de matemáticas. De hecho, siguiendo a Yavuz, Ozyildirim y Doganc (2012) predisponen positivamente al alumnado y, en consecuencia, mejoran sus resultados en las actividades que desarrollan de esta disciplina. Una mejor actitud hacia las matemáticas se corresponde con un menor nivel de ansiedad, mientras que de un mayor grado de estrés se deriva un menor rendimiento académico (Palacios, Hidalgo, Ortega y Maroto, 2013). De este modo, cobra relevancia la inteligencia emocional asociada con el aprendizaje, lo que exige prestar atención a los aspectos relacionales y personales para garantizar, en mayor medida, el éxito académico y cotidiano del alumnado.

Becerra-González y Reidl (2015) consideran que las variantes motivacionales se relacionan con el rendimiento académico; mientras Rosário et al. (2012) sostienen que el rendimiento en la materia de Matemáticas se encuentra afectado por las variables asociadas con: la autoeficacia, el fracaso escolar y la autorregulación del aprendizaje; pudiendo derivarse las anteriores de las de índole motivacional y contextual. Mientras que Rondero y Font (2015) indican que la estructura matemática, a nivel individual, tiene origen en procesos cognitivos relacionados con la vida diaria.

Cabe resaltar que ante el poderoso papel que las emociones ocupan en el proceso de enseñanzaaprendizaje es fundamental que el profesorado profundice en este aspecto asociándolo con el desarrollo de competencias para el ejercicio profesional (Hoy, 2013). En este sentido, Lumpe, Czerniak, Haney y Beltyukova (2012) consideran que los aspectos emocionales vinculados con el proceso formativo deben ser conocidos y valorados por los técnicos de la educación. Flores y Gómez (2010) demandan sutilmente del profesorado mecanismos que permitan influenciar a los estudiantes para mejorar la motivación con el objeto de incrementar su nivel de autonomía. Con todo, cabe traer a colación que el uso de un tipo u otro de estrategias de enseñanza presenta efectos relevantes al dinamizar la actividad académica de los estudiantes, contenerla o frenarla.

Se tiene constatado que la motivación aumenta en el alumnado cuando se incrementa el placer que sienten con las actividades desarrolladas en la asignatura de matemáticas y que, además, mejora su éxito escolar (Lim y Chapman, 2013). De hecho, es lógico que la motivación se encuentre reforzada cuando se produce una combinación adecuada entre aspectos positivos con diferentes variables. En este sentido, Brophy (2010) afirma que el aprendizaje resulta divertido y excitante, por lo menos cuando el currículum está bien relacionado con los intereses del alumnado, es acorde a sus capacidades y el profesorado promueve el desarrollo de actividades prácticas.

Cualquier cambio beneficioso para los resultados de aprendizaje tiene influencia en el alumnado y deriva en emociones positivas; mientras que la ausencia de motivación idónea puede desencadenar el fracaso en los estudiantes y en los propios docentes, al facilitar la creación de condiciones propicias para producir desmotivación, generando un círculo vicioso (Ahmed, Werf, Kuyper y Minnaert, 2013). Para cambiar esto, es importante promover alternativas que posibiliten al profesorado repensar el proceso de enseñanzaaprendizaje del que son co-participantes, con interés y responsabilidad directa. Cabe resaltar que la investigación sobre la didáctica de las matemáticas presenta una manifiesta dificultad. Con todo, según Romera-Iruela (2011), los docentes cuentan con un papel fundamental para favorecer la mejora continua en la educación, vinculando la teoría con su práctica, con la finalidad de modificar la práctica escolar. Este cambio puede, incluso, producirse desde una reflexión sistemática o autocrítica sobre la propia práctica educativa.

Partiendo de la responsabilidad del profesorado, en el diseño de la enseñanza y en su implementación, es importante vislumbrar situaciones que inciten a la reflexión y contribuyan a describir o reconocer los 
puntos fuertes, así como las debilidades de la praxis. Aunque no sea con una finalidad directa, de investigación científica, la tarea de análisis y reflexión continuada, de modo individual o grupal/colegiado por parte de los docentes, es deseable y aconsejable desarrollarlos por motu proprio. De este modo, la promoción de la autorreflexión profesional, a la par de la investigación y divulgación de sus resultados, puede contribuir a detectar lagunas, al tiempo que permite concienciar sobre el interés de aplicar estrategias innovadoras en la materia de matemáticas en la educación secundaria y, en consecuencia, para asentar el uso de buenas prácticas curriculares.

Una vez revisada la literatura científica cabe agregar que resultó oportuno, partiendo de un procedimiento introspectivo, focalizar el problema de investigación en la falta de motivación del alumnado de enseñanza secundaria en la materia de matemáticas. De este modo, la finalidad central del estudio es identificar y analizar, desde la perspectiva del profesorado, el alcance de la desmotivación de los estudiantes de educación secundaria en la materia de Matemáticas; con esta pretensión se consideran los siguientes objetivos específicos: 1) determinar los aspectos que se asocian a la falta de motivación del alumnado; 2) profundizar en las disputas relacionadas con la desmotivación hacia el aprendizaje; 3) revelar las problemáticas con las que se encuentran los docentes ante la falta de motivación de los estudiantes, y 4) descubrir el papel del entorno en la desmotivación por el aprendizaje.

\section{Método}

El estudio presentado se encuadra en la metodología cualitativa, a través de la técnica de la entrevista. Esta modalidad de investigación biográfico-narrativa posibilita el análisis del punto de vista de los implicados. Sus historias y opiniones permiten descubrir el conocimiento producido con múltiples experiencias y distintas voces, que son esenciales para la identificación de problemas (Barrett y Stauffer, 2009). Al tiempo contribuyen a promover la reflexión en los participantes a través de su introspección. Además, la investigación cualitativa permite analizar el contexto objeto de estudio facilitando su visibilización.

Participantes. Como es habitual en la investigación cualitativa, para acotar la muestra no se estableció con anterioridad el número de participantes. Se dejó este aspecto sin determinar, en un primer momento, con el objeto de delimitarlo en función de la saturación de los datos o de los resultados obtenidos (Miles y Huberman, 1984). Además, la selección de los docentes no obedece a criterios probabilísticos ni busca la representatividad estadística de la población. Por ello, se partió de la disponibilidad del profesorado que, después de ser informado sobre los objetivos del estudio y asegurándole el anonimato, decidió colaborar libremente.

A partir de las condiciones referidas se entrevistó a 70 profesores de matemáticas en la enseñanza secundaria de Portugal, 51 mujeres y 19 hombres. Como puede observarse, la participación por género es desequilibrada, en parte es consecuencia de la realidad, el número de profesoras es mucho mayor en la población de docentes de enseñanza secundaria (Direção-Geral de Estatísticas da Educação e Ciência [Dirección General de Estadística, Educación y Ciencia, DGEEC], 2013). El profesorado participante procede de 30 escuelas públicas, dispersas en el norte de Portugal, en un total de 21 municipios. En la mayoría de los casos la participación por escuela no es numerosa.

En lo relativo a la edad de los entrevistados, el grupo más numeroso se ubicó entre 41 y 50 años, y más de un tercio entre 31 y 40 años; en menor proporción contaban con más de 50 años y una de las profesoras con 28. En cuanto a la experiencia docente en la materia de matemáticas se determinó por el tiempo de servicio de la mitad de los participantes (más de 20 años de ejercicio profesional), mientras alrededor de un tercio contaba entre 11 y 20 años, y un pequeño grupo ha trabajado 10 años o menos.

Todo el profesorado, que colaboró en el estudio estaba ejerciendo en el sistema de enseñanza público portugués: 9 en régimen de contrato (7 mujeres y 2 hombres); y 61 con vinculación permanente (44 mujeres y 17 hombres). De modo que la mayoría de estas profesoras, con una amplia experiencia docente (más de 11 años de servicio) pertenece al cuadro de personal del Ministerio de Educación portugués. 
Elaboración del instrumento y recogida y análisis de datos. Se optó por la recogida de información a través de la entrevista, realizada a partir de un guion de preguntas estructuradas, con el objetivo de conocer la percepción del profesorado sobre el tópico de estudio. El instrumento fue elaborado ad hoc para responder a los objetivos de la investigación. Para su construcción se contó con el asesoramiento de 7 expertos en metodología cualitativa y del área de didáctica de las matemáticas. Además, se efectuó la validación del instrumento por los mismos investigadores. Por último se sometió el guion de la entrevista a revisión para su aprobación por la DGEEC (dependiente del Ministerio Portugués), que ha subvencionado la investigación.

Para la recogida de los datos fueron necesarios seis meses, y se concretó con el profesorado a partir de un primer contacto establecido por las investigadoras con los respectivos entrevistados. La duración de las sesiones de las entrevistas osciló entre 40 y 70 minutos. Posteriormente los testimonios recogidos fueron transcritos en un documento de texto digital, para poder prepararlos para el análisis.

En el análisis de contenidos, aplicado a las entrevistas, se utilizó el software de Analysis of Qualitative Data (AQUAD) versión 6 . El proceso de codificación se desarrolló a partir de la información bruta obtenida con las narrativas, aunque atendiendo a las finalidades de la investigación planteada. El procedimiento de categorización, de acuerdo con Saldaña (2015) funciona con un sistema de códigos que posibilitando la reducción de los datos sirven para obtener los resultados en la investigación cualitativa. Según Zhang y Wildemuth (2009), este tipo de análisis permite condensar la información íntegra en categorías de 1er. nivel y en subcategorías (de 2o., 3er., etc. nivel). Cabe indicar que la categorización inicial (1er. nivel) se determinó a partir de los objetivos de investigación y las preguntas de la entrevista. Al inicio fue necesario profundizar en el contenido de la información recogida y etiquetar las ideas principales como focos significativos de análisis, así se identificaron las categorías primarias y las subcategorías emergieron a partir del conjunto de las respuestas narradas por el profesorado.

El análisis de contenido abordado ha sido realizado por pares de investigadoras, quienes a partir de la información bruta de modo colaborativo han acotado el listado preliminar de las unidades principales de análisis asociadas a los respectivos objetivos del estudio. Posteriormente se debatió ese primer etiquetado con dos expertos y a partir de su asesoramiento se establece el catálogo y la re-definición de las distintas categorizaciones. La fase siguiente se ha centrado en el desarrollo en profundidad del análisis de contenido de las narrativas, por pares de investigadoras. Lo que ha posibilitado aglutinar los códigos en metacódigos que representan las subcategorías y determinar su nivel de preponderancia. El nivel de predominio o singularidad de los resultados se expone icónicamente a través del distinto/similar tamaño que se le otorga a las diferentes figuras. Tratándose de un estudio cualitativo no se pretenden exhibir ni enfatizar a través de parámetros numéricos.

Cabe indicar que la credibilidad de los resultados en la investigación cualitativa está respaldada por la rigurosidad con que se diseñada y desarrolla el proceso de análisis, como ha sido el caso; a la vez, los resultados obtenidos han ganado fiabilidad a partir de la sistematización obtenida con el software de análisis utilizado y el tratamiento de los mismos por pares de investigadoras. La consistencia interna (fiabilidad) de los resultados presentados también se ve reforzada al acompañarlos de algunos extractos de las narrativas de los participantes, que proporcionan evidencias prolijas que invitan a la reflexión, así como a comprender y conocer con mayor rigor y profundidad el objeto de estudio (Klüber y Burak, 2012).

\section{Resultados}

Como se desprende de los resultados obtenidos, el bajo éxito escolar que de forma recurrente se asocia con la materia de matemáticas constituye una preocupación del profesorado, que lo asocia con la falta de motivación del alumnado. Los factores que expresan los docentes sobre la desmotivación escolar de los adolescentes se agruparon en función de dos categorías principales a raíz de las problemáticas asociadas o derivadas del entorno escolar y socio-familiar.

1 Las condiciones sobre los instrumentos de investigación, para la recogida de información en las escuelas, exigidas en Portugal por la normativa en vigor son establecidas por el Ministério da Educação (2007) en el Diário de República II série, n 140. Recuperado de www.dre.pt 


\subsection{Falta de motivación asociada al entorno escolar}

Las distintas razones que, de modo notorio, identifica el profesorado en la desmotivación del alumnado, en la materia de matemáticas, están asociadas al tipo de formación de estos últimos (ver figura 1).

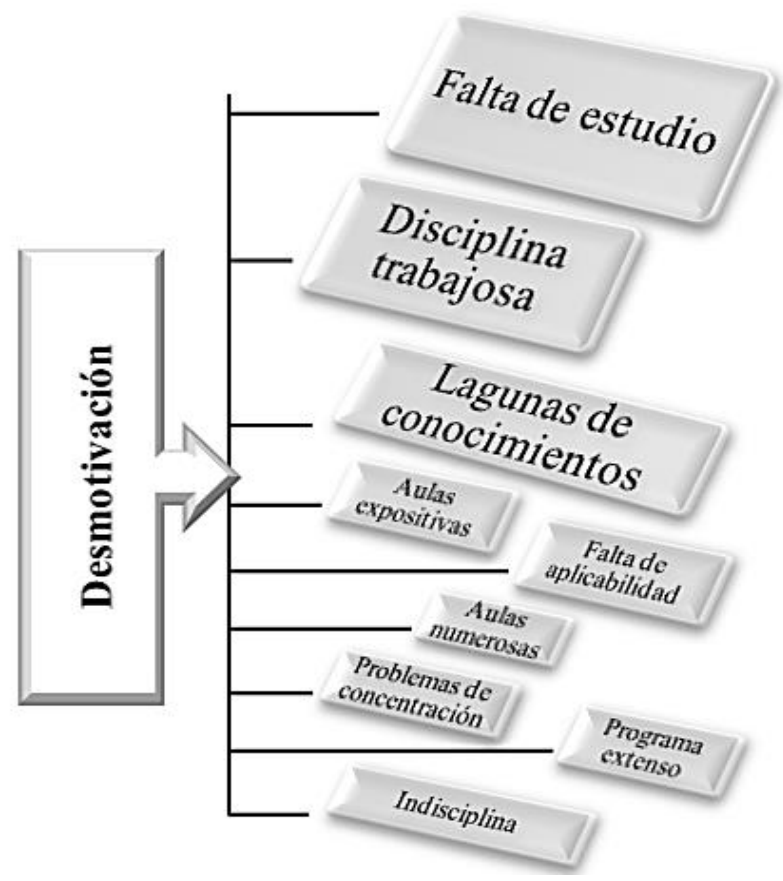

Figura 1. Factores que propician la desmotivación al alumnado en Matemáticas

Los docentes consideran que la desmotivación de los estudiantes en la materia de matemáticas es fruto de: la insuficiente dedicación del alumnado al estudio; las exigencias de la materia, que requiere un importante esfuerzo; lagunas de conocimientos que presenta el alumnado en la disciplina. En contraposición, desde el punto de vista del profesorado, el trabajo desarrollado de forma sistemática es una garantía de éxito escolar; además, consideran que deriva en buenos resultados académicos y, como consecuencia, incrementa la motivación de los estudiantes hacia las matemáticas.

Las lagunas sobre contenidos básicos también son consideradas por parte del profesorado como causa de la falta de motivación en diferentes alumnos. Esto por entender que el sistema de enseñanza se encuentra organizado y se implementa de tal forma que transmite la idea de un conocimiento fragmentado, a lo largo de toda la trayectoria escolar. Además, los docentes reconocen que aplican una evaluación aislada, que se practica materia a materia, sin establecerse una relación global que contribuya a facilitarle al alumnado un sentido pleno para facilitarle la comprensión. El siguiente párrafo ejemplifica esto:

Considero que el alumnado con falta de motivación es el que a lo largo de los años pasados no ha adquirido los conocimientos inherentes y siente dificultad en comprender cada tema, debido a que no se encuentra preparado para las crecientes exigencias que se le plantean (entrevista_17: Profesora con 14 años de servicio).

Más de la mitad de los docentes implicados en el estudio justifican la falta de motivación en algunos alumnos por el hecho de que la materia les requiere esfuerzo y mucho trabajo. De este modo, entienden que exige una gran implicación, mucho tiempo y la realización de un elevado número de tareas. Además, señalan que los estudiantes precisan de conocimientos básicos sólidos, hábitos de estudio, una buena sistematización en el trabajo individual y un importante esfuerzo que han de conciliar con las restantes asignaturas, así como con las apetencias de la juventud actual. 
Por otra parte, con menor insistencia y argumentos más reducidos el profesorado se refiere a los falsos mitos considerados por el alumnado con relación a la aparente inaplicabilidad de los contenidos de la materia a la vida cotidiana; dificultades de concentración-atención de los estudiantes; repercusión negativa generada por el trabajo realizado con clases numerosas; problemas de indisciplina en el aula provocados por el mal comportamiento de algunos alumnos; extensión excesiva de los programas de la materia de matemáticas; y prevalencia de aulas expositivas.

Excepcionalmente algún docente atribuye la desmotivación de ciertos alumnos en la materia de matemáticas a dinámicas poco ejemplificadoras relacionadas con el inadecuado desempeño profesional. Desde el punto de vista del profesorado, las clases expositivas que con frecuencia se desarrollan inciden de forma negativa en la motivación de los discentes; los docentes reconocen que el uso que realizan de metodologías tradicionales no contribuye a motivar a los estudiantes. Algunos docentes señalan que la falta de motivación está relacionada con los problemas de concentración que afectan al alumnado. Esta interpretación puede ser confundida con uno de los síntomas de la desmotivación, al entender que la falta de atención se origina por no crear las condiciones óptimas para el éxito académico y de forma tendencial desemboca en su desencadenante. De hecho, los problemas de desconcentración pueden ser una consecuencia de la desmotivación. Una profesora considera que: "El alumnado demuestra falta de inversión personal a nivel de concentración académica en las aulas, así como de un trabajo persistente y sistemático en la materia de matemáticas" (Entrevista_57: Profesora con 22 años de servicio).

El profesorado percibe que su dedicación en las tareas escolares, entendiendo ésta como requisito para el éxito escolar, no está al alcance de sus posibilidades, no responde a sus expectativas y produce desmotivación en los adolescentes. Por otra parte, reconocen que la materia de matemáticas contiene un fuerte componente secuencial y abstracto; en consecuencia, argumentan que los contenidos impartidos que deben ser interiorizados por el alumnado exigen razonamiento lógico, comprensión e inteligencia. Cuando en este proceso los estudiantes encuentran dificultades perciben que también tienden a desmotivarse.

En la última década, algunos posicionamientos y hechos pudieron haber transmitido la idea de que al consentir la legislación educativa, con el paso de curso o de etapa (en Portugal está permitido realizar el cambio de etapa con 2 o menos asignaturas), se propicia una falta de exigencia de los profesionales que, además, en opinión de distintos entrevistados, de manera paulatina incrementa la desmotivación en el alumnado.

Por otro lado, el colectivo analizado sostiene que los estudiantes, al no ser capaces de relacionar lo que aprenden con el mundo real o con su futuro profesional, no consideran necesario el esfuerzo en la materia de matemáticas (al no verle utilidad); en este sentido, una de las entrevistadas comenta:

La poca relación de la materia de matemáticas con la vida cotidiana, del día a día, dificulta la abstracción. Además, sumándole lo poco atractivo que son ciertos temas es normal que se genere desmotivación en el alumnado por la materia de matemáticas (entrevista_63: Profesora con 8 años de servicio).

Desde la percepción de distintos docentes las clases numerosas impiden desarrollar o aumentar la motivación del alumnado, por el hecho de no reunir o impedir el avance de condiciones propicias para un acompañamiento didáctico individual en el aula. En consecuencia, consideran que el alumnado no puede beneficiarse suficientemente de las explicaciones del profesorado y, por consiguiente, se distraen en mayor medida.

Algunos entrevistados contemplan la poca exigencia del contexto escolar como promotora de la desmotivación. En este sentido, afirman que la carencia o escasez de motivación repercute en el nivel de adquisición de los contenidos matemáticos y en la adquisición de comportamientos que, por sí mismos, pueden originar situaciones poco adecuadas para incrementar el éxito escolar. Por consiguiente, mantienen que ante el bajo rendimiento la desmotivación aflora en los adolescentes o se incrementa de forma notoria y rápida. 
Otro factor con el que los entrevistados justifican la falta de motivación deriva de la exigencia que los propios docentes presentan al tener que implementar programas académicos demasiado extensos, que les predisponen a una menor dedicación, en las explicaciones, y también a un peor acompañamiento didáctico personalizado. Asimismo, declaran que en algunas ocasiones deben impartir los contenidos con demasiada rapidez, impidiendo que los estudiantes dispongan del tiempo necesario para la interiorización del aprendizaje y en determinadas situaciones hay contenidos que no está previsto abordar de nuevo en los siguientes cursos, comprometiendo con ello el desarrollo de competencias importantes, lo que repercute también de forma negativa en la motivación.

Para un pequeño número de profesores la indisciplina propicia la falta de motivación y la relacionan con el síntoma del propio problema. De hecho, el profesorado concibe el mal comportamiento como propulsor del fracaso escolar eventual o permanente y, en consecuencia, de la desmotivación crónica, al menos en buena parte del alumnado. Los docentes manifiestan una actitud comprensiva ante la desmotivación de algunos alumnos en la materia de matemáticas, asumiendo que la falta de éxito académico le genera desánimo ante el aprendizaje. Cabe resaltar que todo el profesorado entrevistado aportó argumentos sólidos sobre la desmotivación de los adolescentes en la materia de matemáticas y sólo una parte los relacionó con el fracaso académico que se produce en esta asignatura; el profesorado señaló que los estudiantes exteriorizan este sentimiento a través de comportamientos impropios.

\subsection{Falta de motivación atribuida al ambiente socio-familiar}

Un amplio grupo de docentes apunta distintos argumentos sobre la desmotivación en la materia de matemáticas ligados con las experiencias del alumnado en el entorno familiar y social, que se producen por: escasa persistencia; creencias previas inapropiadas para enfrentarse al estudio; bajo nivel de exigencia familiar; falta de o escasas expectativas; intereses divergentes a los de la escuela; contexto social perjudicial (ver figura 2).

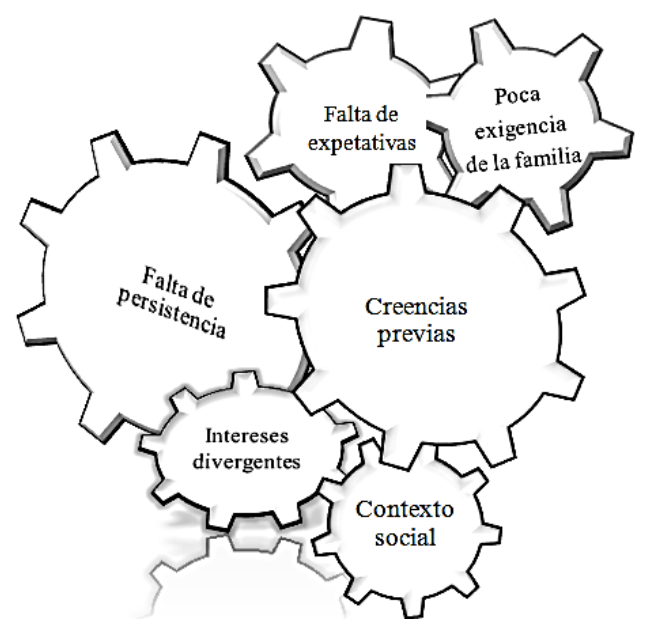

Figura 2. Desmotivación asociada al entorno del alumnado

El profesorado considera la poca exigencia que marca el entorno familiar como un desencadenante de la desmotivación de los estudiantes, con repercusión directa sobre el proceso de aprendizaje. En concreto, entiende que la familia no les inculca una cultura de esfuerzo basada en el trabajo, la perseverancia y la dedicación a la tarea. El siguiente comentario ilustra esto: "La ausencia de responsabilidad, instalada en muchas familias, dificulta la efectiva rentabilidad de los recursos disponibles por el sistema educativo para mejorar la competencia matemática de nuestros adolescentes" (Entrevista_26: Profesor con 21 años de servicio). 
Los docentes opinan que el panorama desmotivador es reflejo del ambiente en el que los adolescentes se encuentran inmersos. En general coinciden en que el alumnado invierte poco tiempo para adquirir autonomía en el estudio y regularse ante el trabajo académico. Algunos docentes apuntan que la falta de persistencia es uno de los principales aspectos que le produce la desmotivación, consideran que ante la adversidad los estudiantes no son capaces de poner en funcionamiento estrategias de resolución de problemas. De este modo, perciben la falta de horizontes del alumnado, en paralelo a las dificultades que encuentra, como desencadenante del fracaso académico en matemáticas, y manifiestan preocupación por la actitud poco apropiada de muchos escolares:

Actualmente, de forma generalizada, el alumnado está habituado a que todo les sea facilitado, cuando le surge un contratiempo no siente necesidad de esforzarse para resolverlo, le resulta más fácil y práctico pedir a alguien que se lo solucione. Asimismo, la falta de persistencia es el principal obstáculo para obtener éxito en una materia con cierta complejidad, como la de matemáticas (Entrevista_64: Profesora con 13 años de servicio).

Los intereses opuestos a los de la institución escolar que presentan en ocasiones los adolescentes es otro de los problemas que percibe el profesorado. Los profesores indican que muchos incentivos que rodean a los estudiantes les resultan con más atractivos que el estudio, y muchos de ellos se contraponen a los intereses o exigencias escolares. Desde la perspectiva de los docentes los estudiantes no son capaces de direccionar su esfuerzo para aplicarlo a las actividades escolares y no asocian la repercusión del aprendizaje a la posible mejora de su situación sociocultural y económica.

(...) el alumnado piensa que el estudio implica mucho trabajo y no tiene garantía de un éxito seguro. "¿Para qué voy a estudiar? ¿Para estar desempleado?". Es una frase muy común, principalmente en el alumnado de los cursos de formación profesional que cursan la disciplina de matemáticas (Entrevista_37: Profesor con 22 años de servicio).

Algunos entrevistados argumentaron la falta de motivación de los alumnos debido a causas que derivan del contexto social en el que están inmersos, así como a las creencias originadas y alimentadas por el ambiente. Los docentes entienden que el entorno privilegia aspectos puramente materiales de la vida; en este sentido consideran que muchos alumnos se ven influenciados por el ambiente familiar que por lo general no promueve ni incentiva el gusto por la actividad intelectual y los valores humanos que exigen algún tipo de esfuerzo, entrega o dedicación.

\section{Discusión y conclusiones}

Con relación a los objetivos definidos y a los resultados obtenidos se puede concluir que el profesorado atribuye la falta de motivación en la materia de matemáticas a problemáticas asociadas a los adolescentes, al currículum de la materia y al contexto escolar. Conciben como factores vinculantes la condición de los estudiantes, la participación propia (como docentes), y las características específicas de la materia de matemáticas; así como la fuerte influencia ejercida o producida por el ambiente sociofamiliar de los adolescentes.

El profesorado destaca como focos de atención ligados con la desmotivación del alumnado los relacionados principalmente con: la falta de trabajo académico; tratarse de una asignatura difícil y que es abordada con metodologías tradicionales; el alumnado no cuenta con conocimientos previos suficientes o los tiene poco consolidados. Además, hacen hincapié en la poca perseverancia que los estudiantes manifiestan en el aprendizaje, la escasa exigencia de la escuela, la existencia de clases numerosas, programas extensos, indisciplina escolar y el hecho de que los contenidos de la materia resulten inicialmente de poca aplicabilidad para la vida cotidiana. Cabe señalar que el uso de metodologías anquilosadas, que no promueven la motivación en los adolescentes, se manifiesta también en otros estudios. En este sentido, Paulino y Silva (2011) revelan que en el contexto escolar el alumnado interpreta como repetitivas, monótonas o sin importancia muchas de las tareas propuestas en la materia de matemáticas. 
Cuando se impone una metodología tradicional en la institución escolar lo habitual es que prime la desmotivación en muchos alumnos. Para mejorar esta situación es necesario buscar mayor sintonía entre los contenidos trabajados, a través de las actividades académicas y la realidad de los adolescentes, que contribuya a despertar sus intereses. Rubel, Chu y Shookhoff (2011) subrayan la importancia de relacionar los contenidos aprendidos en la asignatura de Matemáticas con la realidad y, si es posible, más concretamente con la vida cotidiana de los estudiantes. Para ello es preciso que el profesorado sea capaz de darse cuenta de la necesidad de implementar buenas prácticas (Vásquez y Alsina, 2015). En este sentido, es importante que los docentes tomen conciencia de sus limitaciones y se convenzan de la relevancia que tiene mejorar su praxis para facilitar el cambio metodológico motivado. Por otro lado, Díez-Palomar, Menéndez y Civil (2011) encontraron evidencias sobre la importancia que ejerce el contexto en los procesos de aprendizaje. De hecho, la débil participación del alumnado en el proceso formativo está asociada a la falta de relación de las oportunidades de aprendizaje con sus objetivos futuros (Sfard y Prusak, 2005). Cabe señalar que tratándose de alumnado adolescente la implicación y grado de participación en las dinámicas de aula y la distribución de responsabilidades compartidas resultarán previsiblemente motivadoras y beneficiosas para su proceso de aprendizaje.

Los docentes de Educación Secundaria perciben que los aspectos que producen o incrementan la desmotivación en los estudiantes en la materia de Matemáticas, relacionados con el entorno de los estudiantes, están asociados principalmente a: la poca exigencia de la familia; falta de expectativas; creencias previas inapropiadas; y escasa persistencia en el trabajo académico. Sobre este último aspecto, cabe tener en cuenta que en un estudio realizado por Suárez et al. (2011) se indica que cuando la familia favorece o promueve la persistencia de los hijos ante condiciones adversas se produce una influencia en el comportamiento que actúa de manera positiva sobre el proceso de autorregulación del aprendizaje y el rendimiento académico. De este modo, se pone de manifiesto que la clave de la motivación se encuentra ligada con la empatía y el compromiso ejercitado

Investigaciones como la de Rosário et al. (2012) reflejan la relación entre los factores sociales y el entorno con la motivación. De hecho, los contextos sociales privilegiados fomentan buenas condiciones para el éxito escolar y en los más desfavorecidos por lo general existen o emergen diferentes dificultades. En este estudio se evidencia, a partir de la percepción del profesorado, que la desmotivación de los estudiantes que revelan mayor tendencia se agrava con el incremento de situaciones adversas que encuentra en el día a día. Otra justificación para la desmotivación ligada con componentes sociales se debe, según los docentes, a la visión tradicionalista que existe sobre el conocimiento matemático. Cabe recordar que popularmente se tiende a excusar al alumnado ante el fracaso escolar en esta materia, por atribuirle una elevada dificultad.

La influencia de la familia en la motivación y en el aprendizaje de los hijos es un hecho. En concreto, Bolívar (2006) apunta que el ámbito afectivo de la familia constituye un nivel privilegiado para la primera socialización (criterios, actitudes y valores, sentido de la responsabilidad, motivación por el estudio y el trabajo, equilibrio emocional, desarrollo social, etc.). Además, cabe considerar que en ambientes familiares donde se interpreta el fracaso escolar como una fatalidad, el alumnado tiende a entenderlo de forma semejante. Lo contrario también puede ocurrir, ya que los ejemplos de trabajo, esfuerzo y perseverancia tienden a ser imitados.

De este estudio se desprende que los docentes encuentran que su práctica profesional está rodeada de factores que condicionan e influencian la calidad del proceso enseñanza-aprendizaje y que muchos de ellos presentan un difícil control y solución. Con todo, cabe recalcar la necesidad de asumir nuevos retos que contribuyan, en la era digital, a la creación de sinergias y sistemas de fuerza capaces de influenciar positivamente el proceso formativo y regenerar la escasa motivación que presentan los adolescentes ante el aprendizaje en la materia de matemáticas. De hecho, este trabajo revela patrones consistentes sobre la desmotivación del alumnado de educación secundaria en matemáticas, atendiendo aspectos y características personales (del alumnado y profesorado), profesionales, curriculares y contextuales o ambientales. De este modo, se ofrecen evidencias sobre la situación objeto de estudio, que identifican las principales problemáticas asociadas a la desmotivación del alumnado desde la percepción de los docentes. Su consideración será de utilidad a la hora de planificar la enseñanza en la materia de matemáticas en educación secundaria, así como para la implementación del proceso de aprendizaje. 


\section{Referencias}

Ahmed, W., Werf, G. van der, Kuyper, H. y Minnaert, A. (2013). Emotions, self-regulated learning, and achievement in mathematics: A growth curve analysis. Journal of Educational Psychology, 105(1), 150-161. doi:10.1037/a0030160

Barrett, M. y Stauffer, S. (Eds.) (2009). Narrative inquiry in music education: Troubling certainty. Nueva York: Springer.

Becerra-González, C. y Reidl, L. (2015). Motivación, autoeficacia, estilo atribucional y rendimiento escolar de estudiantes de bachillerato. Revista Electrónica de Investigación Educativa, 17(3), 79-93. Recuperado de https://redie.uabc.mx/redie/article/view/664

Bolívar, A. (2006). Familia y escuela: dos mundos llamados a trabajar en común. Revista de Educación, 339, 119-146.

Brophy, J. (2010). Motivating students to learn. Nueva York: Taylor y Francis.

Díez-Palomar, J., Menéndez, J. M. y Civil, M. (2011). Learning mathematics with adult learners: drawing from parents' perspective. Revista Latinoamericana de Investigación en Matemática Educativa, 14(1), 71-94.

Direção-Geral de Estatísticas da Educação e Ciência (2013). Perfil do docente 2011/2012-Matemática. [Perfil del docente 2011/2012-Matemáticas]. Portugal: Autor.

Flores, R. y Gómez, J. (2010). Un estudio sobre la motivación hacia la escuela secundaria en estudiantes mexicanos. Revista Electrónica de Investigación Educativa, 12(1), 1-18.

Gómez-Chacón, I. (2010). Actitudes de los estudiantes en el aprendizaje de la matemática con tecnología. Enseñanza de las Ciencias, 28(2), 227-244.

Hoy, A. (2013). A reflection on the place of emotion in teaching and teacher education. En M. Newberry, A. Gallant y P. Riley (Eds.), Emotion and school: understanding how the hidden curriculum influences relationships, leadership, teaching, and learning. Advances in Research on Teaching (pp. 255-270). Recuperado de https://www.emeraldinsight.com/doi/abs/10.1108/S1479-3687\%282013\%290000018017

Klüber, T. y Burak, D. (2012). About the aims, objects and problems in brazilian research on mathematical modeling in mathematics education. Bolema, 26(43), 883-905.

Lim, S. y Chapman, E. (2013). Development of a short form of the attitudes toward mathematics inventory. Educational Studies in Mathematics, 82(1), 145-164.

Lumpe, A., Czerniak, C., Haney, J. y Beltyukova, S. (2012). Beliefs about teaching science: the relationship between elementary teachers' participation in professional development and student achievement. International Journal of Science Education, 34(2), 153-166.

Miles, M. y Huberman, M. (1984). Qualitative data analysis. Newbury Park, CA: Sage.

Palacios, A., Hidalgo, S., Ortega, T. y Maroto, A. (2013). Causas y consecuencias de la ansiedad matemática mediante un modelo de ecuaciones estructurales. Enseñanza de las Ciencias, 31(2), 93-111.

Paulino, P. y Silva, A. (2011). Knowing how to learn and how to teach motivation: Contributions from selfregulation of motivation to more a effective learning. Procedia - Social and Behavioral Sciences, 29, 656662. 
Romera-Iruela, M. J. (2011). La investigación-acción en la formación del profesorado. Revista Española de Documentación Científica, 34(4), 597-614.

Rondero, C. y Font, V. (2015). Articulación de la complejidad matemática de la media aritmética. Enseñanza de las Ciencias, 33(2), 29-49.

Rosário, P., Lourenço, A., Paiva, O., Rodrigues, A., Valle, A. y Tuero-Herrero, E. (2012). Predicción del rendimiento en matemáticas: efecto de variables personales, socioeducativas y del contexto escolar. Psicothema, 24(2), 289-295.

Rubel, L., Chu, H. y Shookhoff, L. (2011). Learning to map and mapping to learn our students' worlds. Mathematics Teacher, 104(8), 586-591.

Saldaña, J. (2015). The coding manual for qualitative researchers. Los Ángeles: Sage.

Sfard, A. y Prusak, A. (2005). Telling identities: The missing link between culture and learning mathematics. En H. L. Chick y J. L. Vincent (Eds.), Proceedings of the $29^{\text {th }}$ annual conference of the international group for the psychology of mathematics education (pp. 37-52). Melbourne: PME.

Suárez, N., Herrero, E., Gutiérrez, A., Alba, M., Menéndez, R., García, J., Rosario, P. y Pérez, J. (2011). El fracaso escolar en educación secundaria: análisis del papel de la implicación familiar. Magister: Revista de Formación del Profesorado e Investigación Educativa, 24, 49-64.

Vásquez, l. y Alsina, A. (2015). Un modelo para el análisis de objetos matemáticos en libros de texto chilenos: situaciones problemáticas, lenguaje y conceptos sobre probabilidad. Profesorado. Revista de Currículum y Formación del Profesorado, 19(2), 441-462. Recuperado de

http://www.ugr.es/ recfpro/rev182COL1.pdf

Yavuz, G., Ozyildirim, F. y Dogan, N. (2012). Mathematics motivation scale: a validity and reliability. Procedia-Social and Behavioral Sciences, 46, 1633-1638.

Zhang, Y. y Wildemuth, B. (2009). Qualitative analysis of content. En B. Wildemuth (Ed.), Applications of social research methods to questions in information and library science (pp. 308-319). Westport, cT: Libraries Unlimited. 